\title{
Cosmic rays: the centenary of their discovery
}

\author{
- A.D. Erlykin ${ }^{1,2}$ and A.W. Wolfendale ${ }^{2}$ \\ DOI: 10.1051/epn/2012205 \\ — ${ }^{1}$ P.N. Lebedev Physical Institute, Moscow, Russia \\ Email: erlykin@sci.lebedev.ru \\ [ ${ }^{2}$ Durham University, Durham, UK \\ Email: a.w.wolfendale@durham.ac.uk
}

\section{This year marks the Centenary of the discovery of Cosmic Rays, a crucial step in the development of Modern Physics. Here we describe the early days of this literally fantastic discovery.}

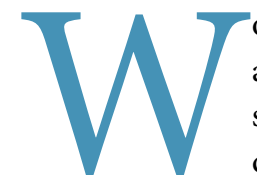

orries about the inability to remove a 'background' reading from electroscopes being used to study the newlydiscovered 'radioactivity' had been around for nearly two decades in 1910. An 'ultra-gamma radiation' was suspected and a number of scientists tried using the atmosphere as an absorber to see the extent to which the radiation fell off with height. By virtue of the combination of an excellent (Wulf) electrometer, a careful and inspired observer (Victor F. Hess), an experienced 'balloon-conductor' and meteorologist, the balloon ascent of August 7, 1912 was successful in pinning down the mysterious radiation. Hess [1] stated that:

'the observations seem to be most readily explained by the assumption that a radiation of very high penetrating power enters our atmosphere from above...'

Peter M. Schuster, President of the Victor Franz Hess Society, has described the circumstances of the successful flight very well:

$\triangle$ Painting by artist Rita Greer (Liss, Hants, UK) based on the famous photograph taken at ground level. Major

Hoffory was the

balloonist and E. Wolf

the meteorologist.
In Aussig upon Elbe, Hess got at his disposal the balloon 'Böhmen', filled with $1680 \mathrm{~m}^{3}$ of hydrogen, and in which he rode off at 06:12 in the morning.

The conductor of the balloon was the well trained Hauptmann (Major) W. Hoffory. D. E. Wolf from the Vienna Central Meteorological Office (Meteorologische Zentralanstalt in Wien) assumed the meteorological observation. Hess himself attended as the observer of the air electrical effects. This had been the seventh balloon ride by Hess, it started at 06:12 on August 7, 1912 and lasted until 12:15 the same day. The previous six balloon rides went off from the Vienna Prater.

The team are to be admired: a hydrogen-filled balloon and rudimentary breathing apparatus (the altitude reached was 17,600 feet) were only two of the hazards.

Figure 1 shows the results from Hess' flights and a confirmation, set by Kolhörster -himself a leader in the field [2]. It is interesting to speculate 'what if'. One such relates to the ionosphere; what if it had been known in 1912, would Hess, an 'atmospheric electrician' have attributed his results to this, later-discovered, phenomenon? We shall never know.

\section{What is the Radiation?}

Understandably the early workers, experienced as they were with 'radioactive' gamma rays, thought that the primaries were gamma rays of some sort, indeed, Millikan clung to this idea for a long time. However, the observation of a latitude effect - a variation of cosmic ray intensity near ground level on latitude, by Clay [3] and others - showed the relevance of the Earth's magnetic field to the cosmic ray trajectories. Charged particles were thus responsible; however the name (cosmic radiation) has stuck to this day. It was 
not until the 1960s with the Explorer II satellite that primary gamma rays as such were detected. Gamma Ray Astronomy is now an important field of study in Astrophysics.

\section{Identifying the Particles}

The romance of 'Cosmic Rays' continued with the detailed study of the particles. The East-West effect [4] indicated that the primaries were mainly positively charged (protons in the main). The use of the cloud chamber (introduced by CTR Wilson, in the 1890s) by skilful operators, particularly Anderson and Blackett and their groups, led to the discovery of muons, positrons and, later, by Rochester and Butler, the 'strange particles'.

The introduction of uniform magnetic fields around the cloud chamber and increasingly complex Geiger counter systems were crucial to the discoveries.

\section{The Positron and the Muon}

Skobelzyn seems to have been the first to recognize a high-energy cosmic ray track in a magnet-cloud chamber [5] (see Figure 2) but pride of place for recognizing the positron goes to Anderson [6]. Fig 3 gives the evidence - surprisingly, a particle travelling upwards but recognized as such by its lower momentum (smaller radius of curvature) above the plate in the middle of the cloud chamber than below it.

An interesting aspect is the relationship of theory to experiment. Did the work of Dirac, in which anti-matter was predicted [7], play any part in Anderson's discovery? Anderson, in a masterly review of the experimental work leading to the claim, [6] gives a resounding 'no'. Anderson also made the statement that the positron could (should?) have been discovered some 3 years earlier by Bothe and Becker.

The muon, too, could have been discovered earlier (by Blackett, and, no doubt others) but pride of place goes to Anderson and Neddermeyer [8]. Once more, superlative cloud-chamber technique was responsible.

The instability of the muon was recognized but, remarkably, the reason for the muon's existence is still not clear. As Rabi famously remarked 'who ordered that?'

Another facet in the interplay (or lack of it) of theory and experiment comes from the story of Yukawa. Some years before the discovery of the muon a particle of somewhat similar mass had been proposed by him [9]. Such a particle (of mass intermediate between that of the proton and of the electron) would be the carrier of the nuclear glue which kept atomic nuclei together.

Fortunately, it seems that most experimenters did not know of Yukawa's work (published in a rarely accessed Japanese journal). The fact that the muon was penetrating and rarely interacting led to its ready acceptance as a different particle when eventually Yukawas particle (the pion) was widely publicised.

\section{Cascade Curves and the Two Cosmic Ray Components}

In the 1930s, many workers had realized that cosmic rays in the atmosphere comprised two components: the hard (mainly muons) and the soft (electrons and gamma rays). Here, theoretical studies were very important, particularly those by Bhabha and Heitler [10] and others. Indeed, it was through such 'theory' that it was possible to identify the different particles.

The first bundles of particles were observed in the 1920s by Skobelzyn in his experiments with the cloud chamber. The study of the complex showers of particles in the lower atmosphere led to the realization by Auger and Maze and others that some such showers had a lateral extent of many hundreds of metres [11]. In turn, the primary energy was shown to be extremely high. Indeed, the maximum energy recorded to date is a little over $10 \mathrm{~J}$, far in excess of the energy yet available at the particle accelerators, although the rate is extraordinarily low, of order one per square $\mathrm{km}$ per century!

\section{The Origin of Cosmic Rays}

Leaving aside such interesting areas as meteorological effects, meteoritic and ice-core studies, the about-tostart field of neutrino astronomy and the possible role of cosmic rays in the origin of life on Earth, we move to theories of origin.

It was apparent to Hess in his early work that the Sun was not the source of Cosmic Rays: there was no nightday difference in their rate although Alfven was vociferous in his claim for a substantial flux from the Sun [12]. Detection of a small spasmodic solar flux was made in the early 1940s.

The apparently isotropic nature of the flux led to the idea of a Galactic or even Universal origin. Millikan's ‘birth cries of atoms in the Universe' was a prominent example of the latter. That a Galactic origin was likely came from

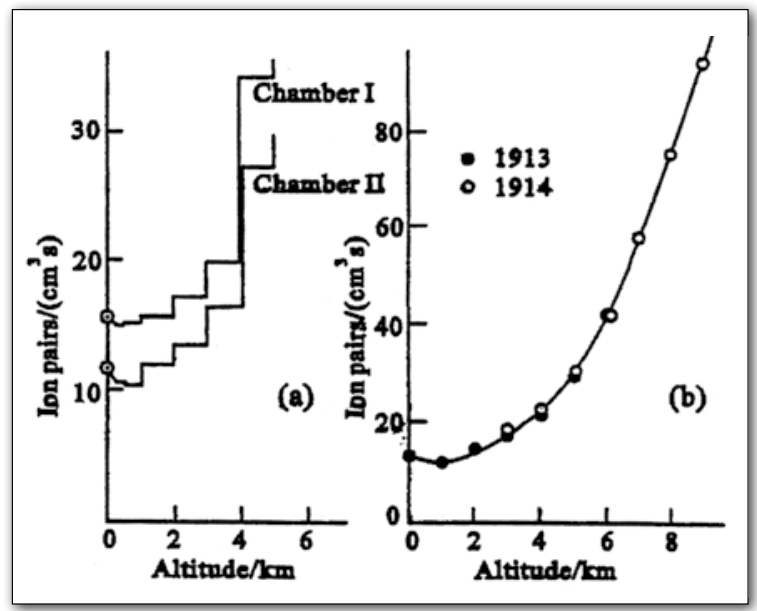

4 FIG 1: Variation of ionization with altitude (a) Hess (1912); (b) Kollhörster $(1913,1914)$ 
